\title{
Source tracking of human leptospirosis: serotyping and genotyping of Leptospira isolated from rodents in the epidemic area of Guizhou province, China
}

\author{
Shijun Li ${ }^{1 *}$, Dingming Wang ${ }^{1}$, Cuicai Zhang ${ }^{2}$, Xiaoyu Wei ${ }^{1}$, Kecheng Tian ${ }^{1}$, Xiuwen Li ${ }^{2}$, Yixin Nie ${ }^{2}$, Ying Liu', \\ Guanghai Yao', Jingzhu Zhou', Guangpeng Tang ${ }^{1}$, Xiugao Jiang ${ }^{2}$ and Jie Yan ${ }^{3,4^{*}}$
}

\begin{abstract}
Background: Sustained human leptospirosis as well as death cases has been reported in Qiandongnan Prefecture, Southeast of Guizhou, China, recently, but these human patients were only clinically diagnosed, and leptospires have never been isolated from patients in these epidemic regions, In order to track the source of infection and understand the etiologic characteristic of leptospirosis, we performed rodent carrier surveillance for leptospirosis in the epidemic area in 2011. The population distribution of rodents in the epidemic regions was revealed.

Results: Four strains of leptospire were isolated from Apodemus agrarius. Microscopic agglutination test (MAT) confirmed the four isolates belonged to leptospiral serogroup Icterohaemorrhagiae. Multilocus sequence typing (MLST) indicated that all the four strains were defined as sequence type 1(ST1), which is identical to the three strains isolated from Rattus tanezumi in Rongjiang County in 2007. Clustering analysis of the MLST data indicated that the local isolates exactly matched with reference strain of leptospiral serovar Lai strain 56601, which is consistent with anti-Leptospira antibody detection of patients using MAT.
\end{abstract}

Conclusions: Apodemus agrarius may be the potentially important carrier of leptospirosis and the potential source of leptospiral infection in human, and serovar Lai maybe the epidemic serovar of Leptospira in the localities.

Keywords: Rodents, Leptospira, Serotyping, Genotyping, Source of infection

\section{Background}

Leptospirosis is one of the most widespread zoonoses and is caused by infection with pathogenic spirochetes of the Leptospira genus [1]. Its incidence in humans is most frequent in developing countries, and the spectrum of human disease ranges from subclinical infection to severe symptoms of multiorgan disfunction with high case fatality rates, reaching mortalities as high as $70 \%$ in the case of severe pulmonary haemorrhage syndrome [2,3]. There is, for certain, an underestimation of the leptospirosis problem

\footnotetext{
* Correspondence: zjumedjun@163.com; med_bp@zju.edu.cn

'Institute of Communicable Disease Control and Prevention, Guizhou Provincial Centre for Disease Control and Prevention, 73 Bageyan Road, Guiyang 550004, Guizhou, People's Republic of China

${ }^{3}$ Department of Medical Microbiology and Parasitology, College of Medicine, Zhejiang University, Hangzhou, People's Republic of China

Full list of author information is available at the end of the article
}

due to a lack of awareness and under-recognition through a lack of proper use of diagnostic tools [4].

Leptospira are maintained in the genital tract and renal tubules of wild and domestic animals and are excreted with urine into the environment where they can survive for several months depending on favorable conditions such as warm, humid environment with a neutral to slightly alkaline $\mathrm{pH}[5,6]$. Rodents are recognized as important mammal reservoirs of Leptospira spp [7,8], which only present mild chronic disease or are asymptomatic, and shed infectious organisms in the urine for their lifetime [9]. Humans may be infected indirectly from animals by contact with contaminated water, soil or mud in a moist environment, or by direct contact with urine, fresh carcasses or organs [10]. Therefore, surveillance on carrier status of reservoir hosts and analysis on the characteristic

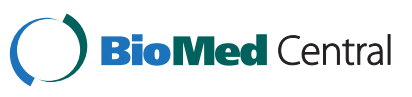


of causative agents contribute to the clinic laboratory diagnosis, active surveillance, outbreak investigation and source tracking for leptospirosis.

Sustained human leptospirosis as well as death cases has been reported in Qiandongnan Prefecture, such as Jinping, Liping, and Rongjiang County, Southeast of Guizhou, in recent years [11]. According to the China National System for Disease Control and Prevention, twelve human leptospirosis cases with one death case were reported in Guizhou in 2011. However, Leptospira were never isolated from patients in recent years and the patients were only serologically diagnosed, and the etiologic characteristics of epidemic Leptospira remain unclear.

Traditionally, pathogenic Leptospira are classified into more than 200 serovars based on serological methods [12]. Nowadays, multilocus sequence typing (MLST) method has been recently proved for typing leptospires [4,13-15]. MLST is a simple PCR based technique, which makes use of automated DNA sequencers to assign and characterize the alleles present in different target genes. The selected loci are generally the housekeeping genes, which evolve very slowly over an evolutionary time-scale $[4,16]$ and hence qualify as highly robust markers of ancient and modern ancestry. The sequencing of multiple loci provides a balance between technical feasibility and resolution.

In order to track the source of infection and understand the etiologic characteristics of human leptospirosis in the epidemic area, we performed rodent carrier status surveillance in Jinping, Liping and Rongjiang County in 2011. Leptospiral isolates were serologically and molecularly identified and typed using MAT and MLST, respectively. Our results will contribute to the prevention and control of leptospirosis in the localities.

\section{Methods}

\section{Rodents collection}

The present study was conducted in three sites including Jinping, Liping and Rongjiang County, where a high number of leptospirosis cases and deaths were reported in recent years.

Rodents were trapped by using Trap-night method [17] (traps with peanut bait) in rice-field environments in Jinping and Liping county, during September to October. Traps were placed at evening and fetched back at the next morning. Trapped rodents were identified by genus, species, and gender based on phenotypic characteristics (ears, body, tail, fur colour and sex) [17]. Rodents were dissected to collect kidneys. Live animals were killed by decapitation under anesthesia by diethyl ether. Kidney tissue samples were collected for isolation and culture of leptospires. Animal protocols were approved by the Animal Ethics
Review Committee of Guizhou Provincial Centre for Disease Control and Prevention.

\section{Leptospiral isolation and cultivation}

Freshly isolated kidney sample were inoculated to $8 \mathrm{~mL}$ liquid Ellinghausen - McCullough - Johnson - Harris $(\mathrm{EMJH})$ medium (Difco, USA) [18]. Cultures were incubated at $28^{\circ} \mathrm{C}$ and evaluated weekly by dark field microscopy for up to 2 months [19]. Leptospira isolates and reference strains belonging to the Chinese 15 serogroups 15 serovars provided by Chinese Centre for Disease Control and Prevention (Chinese $\mathrm{CDC}$ ) were cultivated at $28^{\circ} \mathrm{C}$ in Ellinghausen-McCullough-Johns on-Harris (EMJH) (Difco Laboratories, Detroit, MI, USA) liquid medium supplemented with $8 \%$ heat-inactivated rabbit serum [17].

\section{MAT}

For the serogroup identification of leptospiral isolates, Microscopic agglutination test (MAT) was performed using a battery of anti-serum against the Chinese reference strains belonging to 15 serovars in 15 serogroups provided by Chinese CDC [20]. For detecting antiLeptospira antibodies of serum samples (LCB, LH, ZJD, YCX, LJP, YZM, WSZ, LJX, and LDL) collected from patients in the local regions, MAT was carried using a battery of pathogenic reference strains belonging to Chinese 15 serovars in 15 serogroups of pathogenic Leptospira including leptospiral strains isolated in the epidemic area. The MAT titre was expressed as the reciprocal of the highest serum dilution that resulted in $50 \%$ agglutination of leptospires. The samples with titres $\geq 100$ were recognized as positive.

\section{MLST analysis}

DNA was extracted from cultures of Leptospira strains using DNA Extraction Kit (SBS Genetech, Beijing, China) according to the manufacturer's directions. Seven loci (pntA, sucA, fadD, tpiA, pfkB, mreA, and glmU) were selected based on performance of primers as previously described (also can be obtained from the sharing website: http://leptospira.mlst.net) [21]. Primer sequences are shown in Table 1. Amplifications were performed in $50 \mu \mathrm{l}$ total volumes of PCR reaction system contained approximately $25 \mu \mathrm{l}$ of PreMix Taq (TaKaRa, Otsu, Japan), $2 \mu \mathrm{l}$ of forward and reverse primers with concentrations of $10 \mathrm{pmol} / \mu \mathrm{l}, 2 \mu \mathrm{l}$ of DNA, $19 \mu \mathrm{l}$ of deionized water, respectively. Amplification was performed on an Biometra TProfession thermocycler (Biometra, Goettingen, Germany) using amplification parameters included an initial denaturation at $94^{\circ} \mathrm{C}$ for 5 minutes, followed by 30 cycles of $94^{\circ} \mathrm{C}$ for 10 seconds, $52^{\circ} \mathrm{C}$ (mreA, pfkB, pntA, sucA, and tpiA), or $50^{\circ} \mathrm{C}$ (fadD and glmU) for 15 seconds, $72^{\circ} \mathrm{C}$ for 50 seconds, then $72^{\circ} \mathrm{C}$ for 7 minutes. Following the standard MLST protocol, 
Table 1 Information of loci proposed for MLST of leptospiral isolates

\begin{tabular}{|c|c|c|c|}
\hline Gene & Size of PCR product (bp) & Primer 5'-3' & Annealing temperature $\left({ }^{\circ} \mathrm{C}\right)$ \\
\hline pntA & 638 & $\begin{array}{l}\text { F: TGCCGATCCTACAACATTA } \\
\text { R: AAGAAGCAAGATCCACAACTAC }\end{array}$ & 52 \\
\hline SUCA & 560 & $\begin{array}{l}\text { F: AGAAGAGGCCGGTTATCATCAG } \\
\text { R: CTTCCGGGTCGTCTCCATTTA }\end{array}$ & 52 \\
\hline $\mathrm{pfkB}$ & 560 & $\begin{array}{l}\text { F: CCGAAGATAAGGGGCATACC } \\
\text { R: CAAGCTAAAACCGTGAGTGATT }\end{array}$ & 52 \\
\hline tpiA & 534 & $\begin{array}{l}\text { F: AAGCCGTTTTCCTAGCACATTC } \\
\text { R: AGGCGCCTACAAAAAGACCAGA }\end{array}$ & 52 \\
\hline mreA & 602 & $\begin{array}{l}\text { F: AAAGCGGCCAACCTAACACC } \\
\text { R: CGATCCCAGACGCAAGTAAG }\end{array}$ & 52 \\
\hline$g \operatorname{lmU}$ & 557 & $\begin{array}{l}\text { F: GGAAGGGCACCCGTATGAA } \\
\text { R: TCCCTGAGCGTTTTGATTT }\end{array}$ & 50 \\
\hline $\mathrm{fadD}$ & 577 & $\begin{array}{l}\text { F: AGTATGGCGTATCTTCCTCCTT } \\
\text { R: TTCCCACTGTAATTTCTCCTAA }\end{array}$ & 50 \\
\hline
\end{tabular}

the PCR products were detected by electrophoresis of $1 \mu \mathrm{l}$ of each reaction on a $1.2 \%$ agarose gel for $30 \mathrm{~min}$ at $100 \mathrm{~V}$, and were sequenced by ABI PRISM 377 DNA sequencer. Each allele was assigned a different allele number and the allelic profile (string of seven integers) was used to define the sequence type (ST). A Leptospira mlst website was established to provide public access to these data, and to provide a resource to other investigators who can use this to assign the ST of further strains. This can be accessed at http://leptospira.mlst.net.

\section{Results}

\section{Rodent distribution}

A total of 160 rodents including Apodemus agrarius, Rattus norvegicus, Apodemus chevrieri, Rattus rattus sladerni, Rattus nitidus, Hodgson, Rattus flavipectus, and other rodents were trapped, and the prevalent rodent for Jinping and Liping was Apodemus agrarius, with $37.8 \%$ of the total rodents for Jinping and $21.9 \%$ for Liping, while no
Apodemus agrarius was trapped in Rongjiang County, in which Apodemus chevieri was the prevalent rodents (54.8\%) (Table 2).

\section{Carrier status of rodents}

Three strains of spirochetes (nominated as JP13, JP15 and JP19) were isolated from Apodemus agrarius in Jinping County, with positive rates of $6.7 \%$ (3 strains isolated from 45 rodents), and one strain (nominated as LP62) from Apodemus agrarius in Liping County, with positive rates of $1.4 \%$ ( 1 strain isolated from 73 rodents). No spirochetes were isolated from the sites in Rongiang County. All the other species of rodents were negative for spirochete isolation, except for Apodemus agrarius in the three sites. The positive isolation rates of spirochete from Apodemus agrarius was 17.65\% (3 strains isolated from 17 Apodemus agrarius) for the site in Jingping, and 6.25\% (1 strain isolated from 16 Apodemus agrarius) for the site in Liping (Table 2).

\section{Table 2 Rodent distribution and leptospiral carrier status in the epidemic area of Guizhou Province}

\begin{tabular}{|c|c|c|c|c|}
\hline \multicolumn{2}{|c|}{ Distribution of rodents and statistics of rodent surveillance } & \multicolumn{3}{|c|}{ Data of rodents for the three sites } \\
\hline & & Jinping & Liping & Rongjiang \\
\hline \multirow[t]{7}{*}{ Distribution of rodents } & Apodemus agrarius & $17^{*}$ & $16 \#$ & 0 \\
\hline & Rattus norvegicus & 2 & 2 & 0 \\
\hline & Apodemus chevrieri & 3 & 40 & 20 \\
\hline & Rattus tanezumi & 13 & 3 & 0 \\
\hline & Rattus nitidus Hodgson & 3 & 0 & 0 \\
\hline & Rattus flavipectus & 1 & 4 & 11 \\
\hline & Other rodents & 6 & 8 & 11 \\
\hline \multirow[t]{5}{*}{ Statistics of rodent monitoring } & Number of traps (NT) & 900 & 600 & 600 \\
\hline & Number of trapped rodents (NR) & 45 & 73 & 42 \\
\hline & Percentage of rodents density (NR/NT) & 5 & 12.7 & 7 \\
\hline & Number of isolated strains (NS) & 3 & 1 & 0 \\
\hline & Percentage of positive isolation (NS/NR) & 6.7 & 1.4 & 0 \\
\hline
\end{tabular}

\footnotetext{
* Three strains of leptospire were isolated from seventeen Apodemus agrarius.
}

\# One strain of leptospire was isolated from sixteen Apodemus agrarius. 
Results of serogroup identification of leptospiral isolates MAT was performed using a battery of anti-serum against the Chinese reference strains belonging to 15 serovars in 15 serogroups. All the four strains agglutinated with antiserum against reference strain 56601 belonging to serovars Lai of serogroup Icterohaemorrhagiae with titres $\geq 100$, and no positive results of MAT were observed with antiserum against to strains belong to the other serogroups (Table 3), according to the determine standard that samples with titres $\geq 100$ were recognized as positive.

\section{MLST pattern of leptospiral isolates}

Seven MLST loci based primers were used to amplify the chromosome DNA of leptospiral isolates, and all of the seven loci were successfully amplified from the four isolates. The MLST pattern showed that the four isolates produced a same size of PCR segment at the same locus (Figure 1).

\section{ST of leptospiral isolates}

Seven loci (pntA, sucA, fadD, tpiA, pfkB, mreA, and $\mathrm{glmU}$ ) of the chromosome DNA of the four leptospiral isolates were successfully sequenced. The sequences were analysed following the standard MLST protocol which can be accessed at http:// leptospira.mlst.net, an allele number was assigned to all the allele of different leptospiral strains and the allelic profile (string of seven integers) was defined as sequence type 1 (ST1: 1-1-1-11-1-1) (Figure 2). According to the ST profile, all of the three leptospiral strains exactly matched with Leptospira serogroup Icterohaemorrhagiae Serovar Lai strain 56601.

\section{Clustering analysis results}

Clustering analysis (Figure 2), based on the MLST data of the four leptospiral strains isolated from Apodemus agrarius in 2011, three strains isolated in 2007 and ten reference strains representative to the ten common epidemic serogroups provided by Chinese CDC, suggested that all the 7 leptospiral isolates and reference strain 56601 formed one clade, which indicated that all the leptospiral isolates including four strains isolated from Apodemus agrarius in 2011 and three strains isolated from Rattus tanezumi in 2007 are typed as Leptospira serogroup Icterohaemorrhagiae Serovar Lai.

\section{Anti-Leptospira antibody of suspected patients}

MAT were performed using serum samples of suspected patients with Chinese reference strains belonging to 15 serovars in 15 serogroups of pathogenic Leptospira species and the four leptospiral isolates. Of the 9 serum samples of patients tested by MAT, 66.7\% (6/9) had agglutinating antibodies against isolate JP13, JP15, JP19 and LP62 and reference strain 56601, but not reference strains belong to other serogroups (Table 4).

\section{Discussion}

The present study performed surveillance on rodent carrier status of Leptospira in the epidemic area in 2011. The population distribution of rodents in the epidemic regions was revealed and four strains of leptospire were isolated from Apodemus agrarius. MAT confirmed the four isolates belonged to leptospiral serogroup Icterohaemorrhagiae. MLST define the four isolated as ST1 and exactly matched with reference strain of

Table 3 Results of MAT identification for leptospires isolated from Apodemus agrarius in Guizhou Province

\begin{tabular}{|c|c|c|c|c|c|c|c|}
\hline \multicolumn{4}{|c|}{ Anti-serum against the Chinese reference strains belonging to 15 serovars in 15 serogroups } & \multicolumn{4}{|c|}{ MAT results (titres) of isolated strains } \\
\hline Anti-Serum No. & Strain & Serovar & Serogroup & JP13 & JP15 & JP19 & LP62 \\
\hline 56601 & Lai & Lai & Icterohaemorrhagiae & $+(1: 800)$ & $+(1: 800)$ & $+(1: 800)$ & $+(1: 400)$ \\
\hline 56602 & M10 & Javanica & Javanica & - & - & - & - \\
\hline 56603 & Lin & Canicola & Canicola & - & - & - & - \\
\hline 56604 & Pishu & Ballum & Ballum & - & - & - & - \\
\hline 56605 & 4 & Pyrogenes & Pyrogenes & - & - & - & - \\
\hline 56606 & $\operatorname{Lin} 4$ & Autumnalis & Autumnalis & - & - & - & - \\
\hline 56607 & Sep-65 & Australis & Australis & - & - & - & - \\
\hline 56608 & Luo & Pomona & Pomona & - & - & - & - \\
\hline 56609 & $\operatorname{Lin} 6$ & Linhai & Grippotyphosa & - & - & - & - \\
\hline 56610 & P7 & Hebdomadis & Hebdomadis & - & - & - & - \\
\hline 56612 & L37 & Paidjian & Bataviae & - & - & - & - \\
\hline 56613 & $65-52$ & Tarassovi & Tararrovi & - & - & - & - \\
\hline 56615 & L 105 & Cingshui & Manhao & - & - & - & - \\
\hline 56635 & L 138 & Sejroe & Wolffi & - & - & - & - \\
\hline 56655 & Nan 10 & Mini & Mini & - & - & - & - \\
\hline
\end{tabular}

+: Positive; -: Negative. 


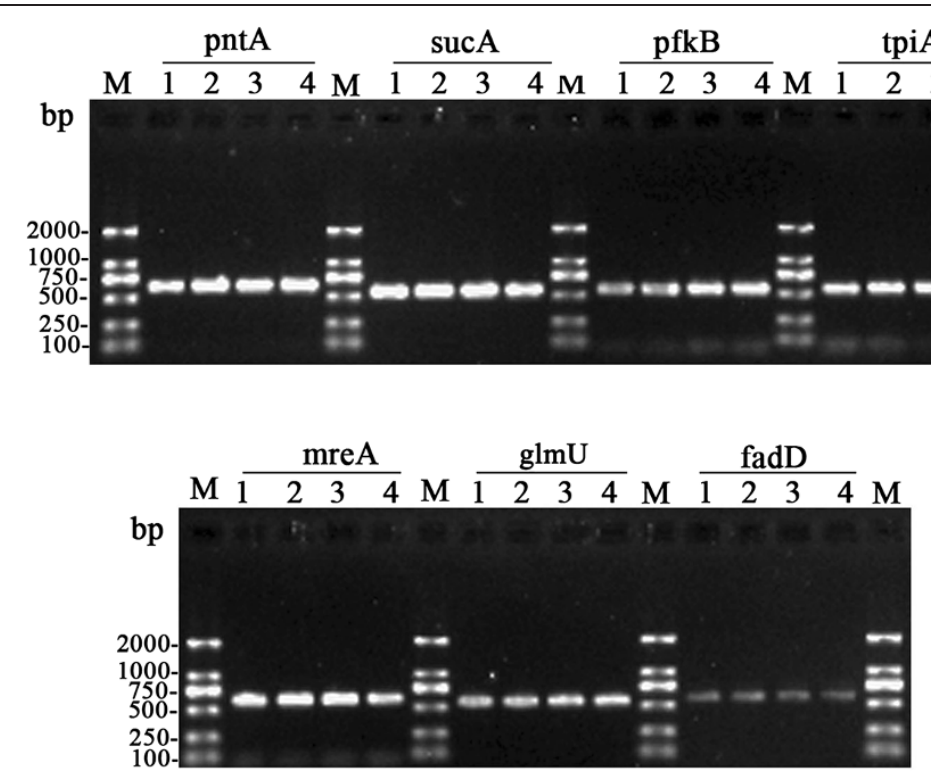

Figure 1 PCR products from the seven selected MLST loci of four leptospiral strains isolated from Jinping and Liping County, Guizhou province. PCR products were electrophoresised through a 1.2\% agarose gel. M: 100 bp DNA Ladder; 1, Leptospira isolate JP13; 2, Leptospira isolate JP15; 3, Leptospira isolate JP19; 4, Leptospira isolate JP62.

leptospiral serovar Lai strain 56601, which is consistent with anti-Leptospira antibody detection of patients using MAT. Together, these findings indicate that Apodemus agrarius may be the potentially important carrier of leptospirosis for Jinping and Liping County, and serovar Lai maybe the epidemic serovar of Leptospira in the epidemic area. Our results will contribute to the control and prevention of leptospirosis in the localities.
Guizhou has been proved the old foci of leptospirosis in China [11,22,23]. Qiandongnan Prefecture of Guizhou province was the high-incidence area of leptospirosis in Guizhou Province. For example, 14126 human leptospirosis cases with 534 deaths were reported in Qiannan prefecture from 1958 to 2005. Investigation on the epidemiology of Leptospirosis in Liping county revealed that a total of 127 leptospirosis cases with 28 deaths

\begin{tabular}{|c|c|c|c|c|c|c|c|c|c|c|c|}
\hline $\begin{array}{l}\text { Categorical } \\
\text { MLST }\end{array}$ & & Serogoup & serovar & ST & & & Alle & nur & & & \\
\hline & 56607 & Australis & Australis & 50012 & 3 & 1 & 1 & 5 & 3 & 6 & 2 \\
\hline & - 56609 & Grippotyphosa & Linhai & 92 & 3 & 1 & 2 & 13 & 3 & 6 & 6 \\
\hline & 56603 & Canicola & Canicola & 37 & 3 & 3 & 3 & 2 & 3 & 4 & 5 \\
\hline & 56608 & Pomona & Pomona & 37 & 3 & 3 & 3 & 2 & 3 & 4 & 5 \\
\hline & 56610 & Hebdomadis & Hebdomadis & 37 & 3 & 3 & 3 & 2 & 3 & 4 & 5 \\
\hline & 56612 & Bataviae & Paidjian & 96 & 3 & 3 & 3 & 2 & 3 & 35 & 5 \\
\hline & 56606 & Autumnalis & Autumnalis & 95 & 1 & 12 & 3 & 20 & 3 & 4 & 6 \\
\hline & 56635 & Sejoroe & Wolffi & 97 & 1 & 18 & 1 & 3 & 2 & 4 & 6 \\
\hline & 56601 & Icterohaemorrhagiae & Lai & 1 & 1 & 1 & 1 & 1 & 1 & 1 & 1 \\
\hline & G30 & Icterohaemorrhagiae & Lai & 1 & 1 & 1 & 1 & 1 & 1 & 1 & 1 \\
\hline & G31 & Icterohaemorrhagiae & Lai & 1 & 1 & 1 & 1 & 1 & 1 & 1 & 1 \\
\hline & G32 & Icterohaemorrhagiae & Lai & 1 & 1 & 1 & 1 & 1 & 1 & 1 & 1 \\
\hline & $\mathrm{JP} 13$ & Icterohaemorrhagiae & Lai & 1 & 1 & 1 & 1 & 1 & 1 & 1 & 1 \\
\hline & JP15 & Icterohaemorrhagiae & Lai & 1 & 1 & 1 & 1 & 1 & 1 & 1 & 1 \\
\hline & JP19 & Icterohaemorrhagiae & Lai & 1 & 1 & 1 & 1 & 1 & 1 & 1 & 1 \\
\hline & LP62 & Icterohaemorrhagiae & Lai & 1 & 1 & 1 & 1 & 1 & 1 & 1 & 1 \\
\hline & 56605 & Pyrogenes & Pyrogenes & 76 & 5 & 1 & 1 & 6 & 2 & 3 & 2 \\
\hline
\end{tabular}

Figure 2 Dendrogram of MLST data showing Leptospira isolates and L. interrogans strain Lai clustering together at $100 \%$. 
Table 4 Results of MAT detection for anti-Leptospira antibody of patients from epidemic region

\begin{tabular}{|c|c|c|c|c|c|c|c|c|c|c|c|c|}
\hline \multicolumn{4}{|c|}{ Isolates and reference strains } & \multicolumn{9}{|c|}{ Serum samples of patients and Agglutination results (titres) } \\
\hline NO. & Strain & Serovar & Serogroup & LCB & LH & ZJD & YCX & LJP & YZM & WSZ & LJX & LDL \\
\hline JP13 & $\mathrm{JP13}$ & $1^{*}$ & Icterohaemorrhagiae & $+(1: 800)$ & $+(1: 800)$ & - & - & $+(1: 400)$ & $+(1: 800)$ & $+(1: 800)$ & $+(1: 800)$ & - \\
\hline JP15 & $\mathrm{JP15}$ & $1^{*}$ & Icterohaemorrhagiae & $+(1: 800)$ & $+(1: 800)$ & - & - & $+(1: 400)$ & $+(1: 800)$ & $+(1: 800)$ & $+(1: 800)$ & - \\
\hline JP19 & JP19 & $/^{*}$ & Icterohaemorrhagiae & $+(1: 800)$ & $+(1: 800)$ & - & - & $+(1: 400)$ & $+(1: 800)$ & $+(1: 800)$ & $+(1: 400)$ & - \\
\hline LP62 & LP62 & $\mu^{*}$ & Icterohaemorrhagiae & $+(1: 400)$ & $+(1: 400)$ & - & - & $+(1: 400)$ & $+(1: 400)$ & $+(1: 400)$ & $+(1: 400)$ & - \\
\hline G30 & G30 & $1^{*}$ & Icterohaemorrhagiae & $+(1: 400)$ & $+(1: 400)$ & - & - & $+(1: 200)$ & $+(1: 800)$ & $+(1: 400)$ & $+(1: 400)$ & - \\
\hline G31 & G30 & $/^{*}$ & Icterohaemorrhagiae & $+(1: 400)$ & $+(1: 400)$ & - & - & $+(1: 200)$ & $+(1: 800)$ & $+(1: 400)$ & $+(1: 400)$ & - \\
\hline $\mathrm{G} 32$ & G30 & ${ }^{*}$ & Icterohaemorrhagiae & $+(1: 400)$ & $+(1: 400)$ & - & - & $+(1: 200)$ & $+(1: 800)$ & $+(1: 400)$ & $+(1: 400)$ & - \\
\hline 56601 & Lai & Lai & Icterohaemorrhagiae & $+(1: 800)$ & $+(1: 800)$ & - & - & $+(1: 400)$ & $+(1: 800)$ & $+(1: 800)$ & $+(1: 400)$ & - \\
\hline 56602 & M10 & Javanica & Javanica & - & - & - & - & - & - & - & - & - \\
\hline 56603 & Lin & Canicola & Canicola & - & - & - & - & - & - & - & - & - \\
\hline 56604 & Pishu & Ballum & Ballum & - & - & - & - & - & - & - & - & - \\
\hline 56605 & 4 & Pyrogenes & Pyrogenes & - & - & - & - & - & - & - & - & - \\
\hline 56606 & $\operatorname{Lin} 4$ & Autumnalis & Autumnalis & - & - & - & - & - & - & - & - & - \\
\hline 56607 & Sep-65 & Australis & Australis & - & - & - & - & - & - & - & - & - \\
\hline 56608 & Luo & Pomona & Pomona & - & - & - & - & - & - & - & - & - \\
\hline 56609 & $\operatorname{Lin} 6$ & Linhai & Grippotyphosa & - & - & - & - & - & - & - & - & - \\
\hline 56610 & P7 & Hebdomadis & Hebdomadis & - & - & - & - & - & - & - & - & - \\
\hline 56612 & L37 & Paidjian & Bataviae & - & - & - & - & - & - & - & - & - \\
\hline 56613 & $65-52$ & Tarassovi & Tararrovi & - & - & - & - & - & - & - & - & - \\
\hline 56615 & L 105 & Cingshui & Manhao & - & - & - & - & - & - & - & - & - \\
\hline 56635 & L 138 & Sejroe & Wolffi & - & - & - & - & - & - & - & - & - \\
\hline 56655 & Nan 10 & Mini & Mini & - & - & - & - & - & - & - & - & - \\
\hline
\end{tabular}

* MAT cannot identify the isolates to Serovar level; +: Positive; +: Negative.

were reported from 2001 to 2008 [11]. According to the China National System for Disease Control and Prevention, there were several cases of leptospirosis patients as well as death cases were reported in Guizhou Province in every year of recent years. For instance, twelve human leptospirosis cases with one death case were reported in Guizhou in 2011. However, the leptospires were never isolated from human and animal in recent years, the reason for the failure of pathogen isolation maybe the using of antibiotics for treatment before collecting samples such as urine and blood from patients, or there is, for certain, an underestimation of the leptospirosis problem due to lack of awareness or experiences, so, these reported cases were only clinically diagnosed, and the source of infection and the characteristic of pathogen remain unclear.

In order to track the source of human leptospirosis, we chose three sites located in Jingping, Liping and Rongjiang County, respectively, the high incidence county of human leptospirosis, to perform surveillance on carrier status of Leptospira in rodents which has been proved as the important mammal reservoirs of Leptospira spp. [7,8]. Four leptospires were isolated from Apodemus agrarius, which is consistent with previous study that the Apodemus agrarius was a very important reservoir host of leptospirosis in Guizhou province. To understand the etiologic characteristic of the isolates, both the classic MAT methods and the molecular method (MLST) which is demonstrated suitable for the typing of common epidemic leptospires in China [21] were applied to identify the four isolates. MAT indicated that the four isolates belonged to leptaspiral serogroup Icterohaemorrhagiae, while MLST revealed the four isolates exactly matched with Serovar Lai strain 56601 belonging to serogroup Icterohaemorrhagiae and the result of MAT was consistent with that of MLST.

To establish a linkage of the isolates with the patients in the epidemic area as well as to give a laboratory evidence for the diagnosis of leptospirosis in the patients, serum samples were collected from patients in the epidemic area for the detection of anti-Leptospira antibody using MAT, the results showed that $66.7 \%(6 / 9)$ of the serum samples of patients had agglutinating antibodies against isolate JP13, JP15, JP19 and LP62 isolates and reference strain 56601, but not reference strains belong to other serogroups, which is consistent with the typing results of leptospiral isolates. It implies that Apodemus agrarius may be the main carrier of Leptospira in Jinping 
and Liping County, serovar Lai maybe the cause for the human leptospirosis in the epidemic area in Guizhou province.

\section{Conclusion}

Rodent carrier surveillance for leptospirosis was performed in the epidemic area of Guizhou in 2011. The results showed that Apodemus agrarius may be the potentially important carrier of leptospirosis and the potential source of leptospiral infection in human, and serovar Lai maybe the epidemic serovar of Leptospira in the localities.

\section{Competing interests}

The authors declare that they have no competing interests.

\section{Authors' contributions}

SL executed the Leptspiral isolation, MAT, PCR and MLST experiments, analyzed the data and drafted the manuscript; CZ participated in the analysis of MLST results; DW participated in the study design; XW participated the MLST experiments; KT participated in the rodents Trapping; $X L$ and $X J$ provided the reference strains of $L$. interrogans; $Y N$ provided the rabbit antiLeptospira serum; YL contributed to the culture of leptospiral strains and the MAT experiments; GY and JZ participated in rodents trapping and Leptospira isolation. GT participated in the study design; JY critically revised the manuscript; all authors read and approved the final manuscript.

\section{Acknowledgements}

This work was supported by the Guizhou Province Governor special funds for outstanding scientific and technological talent (Grant No. Guizhou Province, specifical co-word (2010) 90). We acknowledge the contribution of Jingping County CDC, Liping County CDC and Rongjiang CDC for rodent traping.

\section{Author details \\ ${ }^{1}$ Institute of Communicable Disease Control and Prevention, Guizhou Provincial Centre for Disease Control and Prevention, 73 Bageyan Road, Guiyang 550004, Guizhou, People's Republic of China. ${ }^{2}$ National Institute for Communicable Disease Control and Prevention, Chinese Centre for Disease Control and Prevention, 155 Changbai Road, Changping District, 102206 Beijing, People's Republic of China. ${ }^{3}$ Department of Medical Microbiology and Parasitology, College of Medicine, Zhejiang University, Hangzhou, People's Republic of China. 'Laboratory of Microbiology and Parasitology, College of Medicine, Zhejiang University, 388 Yuhangtang Road, Hangzhou 310058, Zhejiang, People's Republic of China.}

Received: 19 December 2012 Accepted: 27 March 2013 Published: 1 April 2013

\section{References}

1. Levett PN: Leptospirosis. Clin Microbiol Rev 2001, 14(2):296-326.

2. Vijayachari $P$, Sugunan AP, Shriram AN: Leptospirosis: an emerging global public health problem. J Biosci 2008, 33(4):557-569.

3. Gouveia EL, Metcalfe J, de Carvalho AL, Aires TS, Villasboas-Bisneto JC, Queirroz A, Santos AC, Salgado K, Reis MG, Ko Al: Leptospirosis-associated severe pulmonary hemorrhagic syndrome, Salvador, Brazil. Emerg Infect Dis 2008, 14(3):505-508.

4. Ahmed N, Devi SM, de Valverde ML, Vijayachari P, Machang'u RS, Ellis WA, Hartskeerl RA: Multilocus sequence typing method for identification and genotypic classification of pathogenic Leptospira species. Ann Clin Microbiol Antimicrob 2006, 5:28.

5. McBride AJ, Athanazio DA, Reis MG, Ko Al: Leptospirosis. Curr Opin Infect Dis 2005, 18(5):376-386.

6. Nalam K, Ahmed A, Devi SM, Francalacci P, Baig M, Sechi LA, Hartskeerl RA, Ahmed N: Genetic affinities within a large global collection of pathogenic Leptospira: implications for strain identification and molecular epidemiology. PLoS One 2010, 5(8):e12637.

7. Guerra MA: Leptospirosis. J Am Vet Med Assoc 2009, 234(4):472-478. 430
8. Meerburg BG, Singleton GR, Kijlstra A: Rodent-borne diseases and their risks for public health. Crit Rev Microbiol 2009, 35(3):221-270.

9. Vinetz JM: Leptospirosis. Curr Opin Infect Dis 2001, 14(5):527-538.

10. Mayer-Scholl A, Draeger A, Luge E, Ulrich R, Nockler K: Comparison of two PCR systems for the rapid detection of Leptospira spp. from kidney tissue. Curr Microbiol 2011, 62(4):1104-1106.

11. Yang KJY, Luo YP, Wu GQ, Yang ZP, Kang ZG: Epidemiology of leptospirosis in Liping county, Guizhou, 2001-2008. Dis Surveill 2009, 24(10):768-769.

12. Morey RE, Galloway RL, Bragg SL, Steigerwalt AG, Mayer LW, Levett PN: Species-specific identification of Leptospiraceae by $16 \mathrm{~S}$ rRNA gene sequencing. J Clin Microbiol 2006, 44(10):3510-3516.

13. Ahmed A, Thaipadungpanit J, Boonsilp S, Wuthiekanun V, Nalam K, Spratt BG, Aanensen DM, Smythe LD, Ahmed N, Feil EJ, et al: Comparison of two multilocus sequence based genotyping schemes for Leptospira species. PLoS Negl Trop Dis 2011, 5(11):e1374.

14. Romero EC, Blanco RM, Galloway RL: Analysis of multilocus sequence typing for identification of Leptospira isolates in Brazil. J Clin Microbiol 2011, 49(11):3940-3942.

15. Caimi K, Varni V, Melendez Y, Koval A, Brihuega B, Ruybal P: A combined approach of VNTR and MLST analysis: improving molecular typing of Argentinean isolates of Leptospira interrogans. Memorias do Instituto Oswaldo Cruz 2012, 107(5):644-651.

16. Enright MC, Spratt BG: Multilocus sequence typing. Trends Microbiol 1999, 7(12):482-487

17. Yalin W, Lingbing Z, Hongliang $Y$, Jianmin $X$, Xiangyan Z, Xiaokui G, Utpal $P$, Jinhong Q: High prevalence of pathogenic Leptospira in wild and domesticated animals in an endemic area of China. Asian Pac J Trop Med 2011, 4(11):841-845.

18. Perez J, Brescia F, Becam J, Mauron C, Goarant C: Rodent abundance dynamics and leptospirosis carriage in an area of hyper-endemicity in New Caledonia. PLoS Negl Trop Dis 2011, 5(10):e1361.

19. Subharat S, Wilson PR, Heuer C, Collins-Emerson JM: Investigation of localisation of Leptospira spp. in uterine and fetal tissues of nonpregnant and pregnant farmed deer. N Z Vet J 2010, 58(6):281-285.

20. Faine SAB, Bloin C, Perolat P: Leptospira and leptospirosis. 2nd edition. Melbourne, Australia: MedSci; 1999.

21. Zhang CCNY, Li XW, Cui ZG, Jiang XG: Application of multiple-locus variable-number tandem repeat analysis (MLVA) for molecular typing of Leptospira interrogans serogroup Icterohaemorrhagiae. Chin J Microbiol Immunol 2009, 29(12):1144-1147.

22. Guo SHDZ, Li JH: Analysis of leptospirosis epidemic in 31 provinces (1991-2005). J Public Health Prevent Med 2006, 6:8-10.

23. Yang M, Mo RJ: Exploration of Space Distribution on Leptospirosis Epidemic Focus with Host Animal. Practical Prevent Med 2007, 14:46-54

doi:10.1186/1471-2180-13-75

Cite this article as: Li et al:: Source tracking of human leptospirosis: serotyping and genotyping of Leptospira isolated from rodents in the epidemic area of Guizhou province, China. BMC Microbiology 2013 13:75.

\section{Submit your next manuscript to BioMed Central and take full advantage of:}

- Convenient online submission

- Thorough peer review

- No space constraints or color figure charges

- Immediate publication on acceptance

- Inclusion in PubMed, CAS, Scopus and Google Scholar

- Research which is freely available for redistribution 